\title{
Isospin Breaking Corrections to the HVP with Domain Wall Fermions
}

\author{
Peter Boyle ${ }^{1}$, Vera Guelpers ${ }^{2, \star}$, James Harrison ${ }^{2}$, Andreas Juettner $^{2}$, Christoph Lehner $^{3}$, Antonin \\ Portelli ${ }^{1}$, and Christopher Sachrajda ${ }^{2}$
}

RBC/UKQCD Collaboration

${ }^{1}$ School of Physics and Astronomy, University of Edinburgh, Edinburgh EH9 3JZ, United Kingdom ${ }^{2}$ School of Physics and Astronomy, University of Southampton, Southampton SO17 1BJ, United Kingdom ${ }^{3}$ Physics Department, Brookhaven National Laboratory, Upton, NY 11973, USA

\begin{abstract}
We present results for the QED and strong isospin breaking corrections to the hadronic vacuum polarization using $N_{f}=2+1$ Domain Wall fermions. QED is included in an electro-quenched setup using two different methods, a stochastic and a perturbative approach. Results and statistical errors from both methods are directly compared with each other.
\end{abstract}

\section{Introduction}

One of the most promising quantities for the search of signatures of physics beyond the Standard Model at low energies is the anomalous magnetic moment of the muon $a_{\mu}$. A comparison of the experimental result with the Standard Model estimate [1] yields a deviation of $\approx 3 \sigma$, which might be a hint of new physics. However, a further investigation requires a reduction of the errors on the experimental and theoretical determinations of $a_{\mu}$. On the theory side, the largest uncertainty arises from the hadronic vacuum polarization (HVP) contribution to $a_{\mu}$. The current most precise determination relies on experimental input from the cross section of $e^{+} e^{-} \rightarrow$ hadrons [2,3]. In the last years a lot of effort has been undertaken to calculate the HVP contribution to $a_{\mu}$ using lattice QCD (see e.g. [4-8]). To be competitive with the result $e^{+} e^{-} \rightarrow$ hadrons a lattice calculation would require an accuracy of $\lesssim 1 \%$. However, these lattice calculations are usually done in the isospin symmetric limit, i.e. treating the up and down quark as being equal. In nature there are two sources of isospin breaking. The bare masses of the up and the down quark are not equal and quarks have electric charges and thus underly QED interactions. The latter effect not only applies to up and down, but to all quark flavors. These effects are expected to be $O\left(\left(m_{d}-m_{u}\right) / \Lambda_{\mathrm{QCD}}\right), O(\alpha) \sim O(1 \%)$ and thus, need to be included in a lattice calculation aiming at a precision of $1 \%$.

The structure of the proceedings is as follows: In section 2 we describe how isospin breaking and QED corrections can be included in lattice calculations and we show results for the HVP in section 3. The results shown in this proceedings are already published in $[9,10]$. Another independent calculation of QED corrections to the HVP for strange and charm quarks has recently be presented in [11].

${ }^{\star}$ Speaker, e-mail: V.M.Guelpers@soton.ac.uk 


\section{Isospin Breaking Corrections on the lattice}

The expectation value of an observable $O$ can be calculated in terms of the Euclidean path integral. The path integral including QED is given by

$$
\langle O\rangle=\frac{1}{Z} \int \mathcal{D}[U] \mathcal{D}[A] \mathcal{D}[\Psi, \bar{\Psi}] O[\Psi, \bar{\Psi}, A, U] e^{-S_{F}[\Psi, \bar{\Psi}, A, U]} e^{-S_{\gamma}[A]} e^{-S_{G}[U]},
$$

with quark fields $\Psi, \bar{\Psi}$, gluon fields $U$ and photon fields $A$. The fermionic action $S_{F}[\Psi, \bar{\Psi}, A, U]$ now also includes couplings of quarks to photons. The non-compact photon action is given by

$$
S_{\gamma}[A]=\frac{1}{4} \sum_{x} \sum_{\mu, \nu}\left(\partial_{\mu} A_{v}(x)-\partial_{\gamma} A_{\mu}(x)\right)^{2}, \quad \text { with } \partial_{\mu} f(x)=f(x+\hat{\mu})-f(x) .
$$

Two methods are commonly used to include QED in the calculation of the path integral (1). A stochastic method [12] using $U(1)$ gauge configurations for the photon fields and a perturbative method [13], where the path integral (1) is expanded in the electromagnetic coupling $\alpha$. The stochastic and perturbative method are explained in detail in subsections 2.1 and 2.2 , respectively.

\subsection{Stochastic Method}

For the stochastic method, it is convenient to use Feynman gauge, due to the simple structure of the photon action. In momentum space, the photon action in Feynman gauge is given by

$$
S_{\gamma, \text { Feyn. }}[A]=\frac{1}{2 N} \sum_{k, \vec{k} \neq 0} \hat{k}^{2} \sum_{\mu}\left|\tilde{A}_{\mu}(k)\right|^{2}
$$

where $\hat{k}=2 \sin \left(k_{\mu} / 2\right), \mathrm{N}$ is the number of lattice sites and $\tilde{A}_{\mu}$ are the photon fields in momentum space. In this study, we work in $\mathrm{QED}_{L}$ [14], i.e. we subtract all spatial zero modes of the photon fields $\tilde{A}_{\mu}\left(k_{0}, \vec{k}=0\right)=0$. To obtain stochastic photon fields, which are distributed according to $\exp \left(-S_{\gamma, \text { Feyn. }}\right)$, one draws Gaussian random numbers with variance $2 N / \hat{k}^{2}$ for the components $\tilde{A}_{\mu}(k)$ of the photon fields in momentum space. After these photon fields have been generated in momentum space, we transform them to position space using a fast Fourier transform.

In this study, we work in the electro-quenched approximation, i.e. we neglect QED effects for the see quarks. This allows to generate photon fields independent of the $S U(3)$ gluon fields. Once the photon fields are generated, they are multiplied to the gluon fields as

$$
U_{\mu}(x) \rightarrow e^{-i e q_{f} A_{\mu}(x)} U_{\mu}(x) .
$$

Calculations of observables then proceed as without QED but using the QCD+QED link variables (4). We average calculations using $+e$ and $-e$ to exactly remove $O(e)$ noise [15].

\subsection{Perturbative Method}

QED can be treated in a perturbative way by expanding the path integral (1) in the electromagnetic coupling [13]

$$
\langle O\rangle=\langle O\rangle_{0}+\left.\frac{1}{2} e^{2} \frac{\partial^{2}}{\partial e^{2}}\langle O\rangle\right|_{e=0}+O\left(\alpha^{2}\right)
$$


where the subscript 0 indicates, that the expectation value $\langle\cdot\rangle_{0}$ is calculated in pure QCD. At $O(\alpha)$ one finds contributions from either two insertions of conserved vector currents $V_{\mu}^{c}$ or one insertion of the tadpole operator $T_{\mu}$

$$
\langle O\rangle=\langle O\rangle_{0}-\frac{\left(e q_{f}\right)^{2}}{2}\left\langle O T_{\mu}(x)\right\rangle_{0} \Delta_{\mu \mu}(0)-\frac{e^{2} q_{f} q_{f^{\prime}}}{2}\left\langle O V_{\mu}^{c}(x) V_{\nu}^{c}(y)\right\rangle_{0} \Delta_{\mu \nu}(x-y)+O\left(\alpha^{2}\right)
$$

In the Feynman gauge and $\mathrm{QED}_{L}$ the photon propagator is given by

$$
\Delta_{\mu v}(x-y)=\delta_{\mu v} \frac{1}{N} \sum_{k, \vec{k} \neq 0} \frac{e^{i k \cdot(x-y)}}{\hat{k}^{2}} .
$$

For the QED correction to meson masses, this gives rise to three different quark-connected diagrams, which are shown in figure 1. Here, we work in the electro-quenched setup, i.e. we do not consider any diagrams, where the photon couples to see quarks.
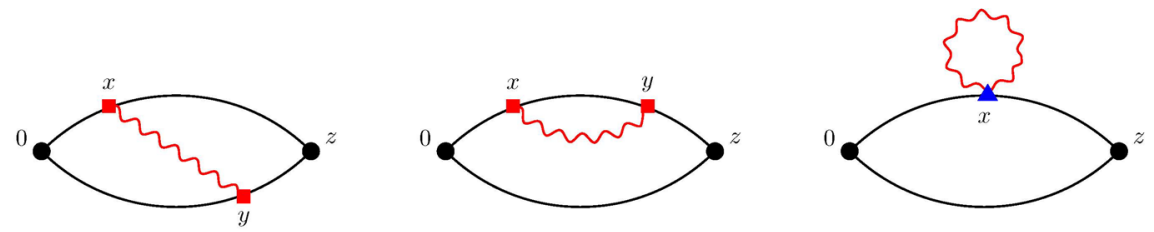

Figure 1. From left to right: Photon exchange diagram, quark self energy diagram and tadpole diagram. Red squared vertices and blue triangle vertices denote insertions of the conserved vector current and the tadpole operator, respectively.

One has to note, that additional diagrams occur, if the operator $O$ in the path integral itself depends on the electromagnetic coupling, as we will see below for the hadronic vacuum polarization.

We calculate the correlation functions required for the diagrams shown in figure 1 in two different ways:

- single- $\mu$ insertion: We start by rewriting the photon propagator by inserting stochastic sources $\eta$

$$
\Delta_{\mu v}(x-y)=\left\langle\sum_{u} \Delta_{\mu v}(x-u) \eta(u) \eta^{\dagger}(y)\right\rangle_{\eta} \equiv\left\langle\tilde{\Delta}_{\mu v}(x) \eta^{\dagger}(y)\right\rangle_{\eta}, \quad\left\langle\eta(u) \eta^{\dagger}(y)\right\rangle_{\eta}=\delta_{u, y} .
$$

This allows to calculate the correlation functions using sequential propagators with insertions of either the conserved vector current $\Gamma_{\mu}^{c}$ and $\tilde{\Delta}_{\mu \nu}(x)$ or $\eta^{\dagger}(y)$. This needs to be done for each combination of the Lorentz indices $\mu, v$ individually and in total requires 17 inversions per quark flavor and source position using Feynman gauge (where only diagonal terms $\mu=\nu$ contribute).

- summed- $\mu$ insertion: Here, we use four different stochastic sources $\xi_{\mu}$ one for each Lorentz index

$$
\Delta_{\mu v}(x-y)=\left\langle\sum_{u} \sum_{\sigma} \Delta_{\sigma v}(x-u) \xi_{\sigma}(u) \xi_{\mu}^{\dagger}(y)\right\rangle_{\xi}=\left\langle\hat{\Delta}_{v}(x) \xi_{\mu}^{\dagger}(y)\right\rangle_{\xi}, \quad\left\langle\xi_{\sigma}(u) \xi_{\mu}^{\dagger}(y)\right\rangle_{\xi}=\delta_{u y} \delta_{\sigma \mu},
$$

and calculate sequential propagators with insertions of either $\sum_{v} \Gamma_{\nu}^{c} \hat{\Delta}_{v}(x)$ or $\sum_{\mu} \Gamma_{\mu}^{c} \xi_{\mu}^{\dagger}(y)$. This requires in total 5 inversions to construct the diagrams given in figure 1.

More details can be found in [10]. 


\subsection{Strong Isospin Breaking}

We account for strong isospin breaking corrections in two different ways, by either using different bare quark masses for the valence up- and down quarks, such that we approximately reproduce the physical quark mass difference from [16] or by an expansion of the path integral in the quark masses as proposed in [17]

$$
\langle O\rangle_{m_{f} \neq \hat{m}}=\langle O\rangle_{m_{f}=\hat{m}}+\left.\left(m_{f}-\hat{m}\right) \frac{\partial}{\partial m_{f}}\langle O\rangle\right|_{m_{f}=\hat{m}}+O\left(\left(m_{f}-\hat{m}\right)^{2}\right),
$$

where $\hat{m}$ is the isospin symmetric quark mass and $m_{f}$ the mass of either the up or the down quark. The derivative in (10) is given by

$$
\left.\frac{\partial}{\partial m_{f}}\langle O\rangle\right|_{m_{f}=\hat{m}}=\langle O \mathcal{S}\rangle_{m_{f}=\hat{m}},
$$

with a scalar current $\mathcal{S}=\sum_{x} \bar{\psi}_{f}(x) \psi_{f}(x)$.

\section{Isospin Breaking Corrections to the Hadronic Vacuum Polarization}

\subsection{Definitions}

We calculate the hadronic vacuum polarization (HVP) tensor from a vector two-point function using a local current at the source and a conserved current at the sink

$$
\Pi_{\mu \nu}(Q)=\sum_{x} e^{-i Q \cdot x} C_{\mu \nu}(x)-\sum_{x} C_{\mu \nu}(x) \quad \text { with } \quad C_{\mu \nu}(x)=Z_{V} q_{f}^{2}\left\langle V_{\mu}^{c}(x) V_{\nu}^{\ell}(0)\right\rangle,
$$

with a zero mode subtraction [18]. We then obtain the HVP form factor $\Pi\left(\hat{Q}^{2}\right)$ from the spatial components of the HVP tensor

$$
\Pi\left(\hat{Q}^{2}\right)=\frac{1}{3} \sum_{j} \frac{\Pi_{j j}(Q)}{\hat{Q}^{2}} \quad \text { with } \quad \vec{Q}=0 .
$$

The QED correction to the local-conserved vector correlation function $C_{\mu \nu}(x)$ receives two contributions. One from the QED correction to the two-point function itself $\delta\left\langle V_{\mu}^{c}(x) V_{\nu}^{\ell}(0)\right\rangle$ and one from the QED correction to the multiplicative renormalisation $Z_{V}$ for the local vector current used in out setup to calculate the HVP

$$
\delta C_{\mu \nu}(x)=\delta Z_{V} q_{f}^{2}\left\langle V_{\mu}^{c}(x) V_{\nu}^{\ell}(0)\right\rangle_{0}+Z_{V}^{0} q_{f}^{2} \delta\left\langle V_{\mu}^{c}(x) V_{\nu}^{\ell}(0)\right\rangle .
$$

When calculating the QED correction to the vector two-point function $\delta\left\langle V_{\mu}^{c}(x) V_{\nu}^{\ell}(0)\right\rangle$ in the perturbative method, one needs to take into account, that the conserved current depends on the link variables, and thus, when including QED, it depends on the electromagnetic coupling. This gives rise to two additional diagrams from the expansion of the conserved vector current at the sink. These diagrams are shown in figure 2.

\subsection{Setup of the Calculation}

For this study, we use $N_{f}=2+1$ dynamical flavors of Domain Wall fermions on a $24^{3} \times 64$ lattice [19] with inverse lattice spacing of $1.78 \mathrm{GeV}$. We use different values for the valence up- and down-quark mass, where we keep the up-quark mass at the mass of the sea quarks $a m_{u}=0.005$ and increase the down-quark mass to $a m_{d}=0.005915$ such that we approximately reproduce the physical mass difference from [16]. For the valence strange-quark mass we use $a m_{s}=0.03224$ [20], which corresponds 

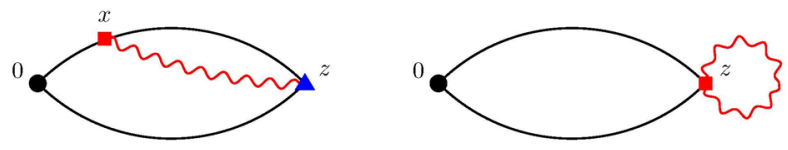

Figure 2. Additional diagrams for the $O(\alpha)$ QED corrections to the HVP from expanding the conserved vector current in the conserved-local vector two-point function.

to the physical strange quark mass without QED. The strange sea-quark mass is $a m_{s}=0.04$. The isospin symmetric pion mass on this ensemble is $m_{\pi} \approx 340 \mathrm{MeV}$. We use 87 gauge configurations and 16 source positions for the quark propagators. For the stochastic method we use one $U(1)$ gauge configuration per $S U(3)$ gauge configuration. For the perturbative method we use one stochastic source $\eta$ for the single- $\mu$ insertion and one set of sources $\xi_{\mu}$ with $\mu=1,2,3,4$ for the summed- $\mu$ insertion per gauge configuration and source position.

\subsection{Results - QED corrections to the vector two-point function}

In figure 3 the QED correction to the HVP from the QED correction to the vector two-point function is plotted against $Q^{2}$. Red squares and blue circles show results from the perturbative and the stochastic method respectively.
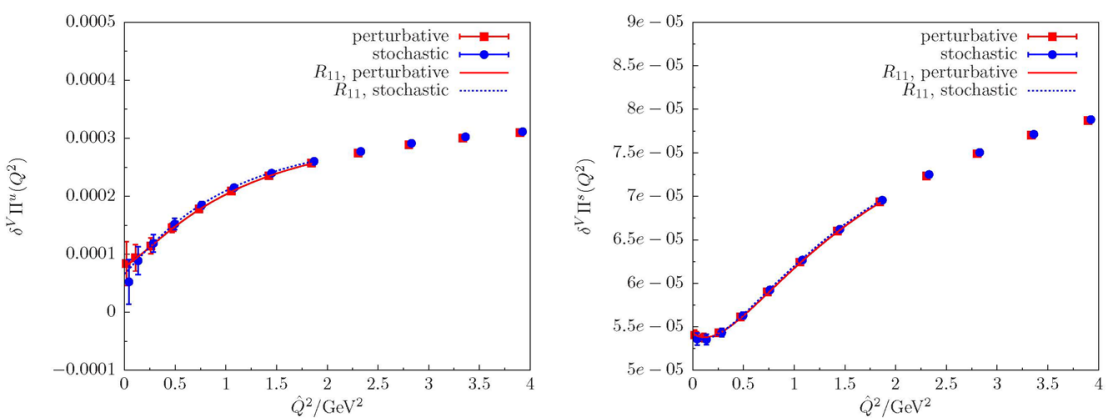

Figure 3. QED correction to $\Pi\left(\hat{Q}^{2}\right)$ from the QED correction to the vector two-point function for the up quark (left) and the strange quark (right).

To extrapolate to $Q^{2}=0$, we use a Padé ansatz, where we allow each parameter in the Padé to receive a $\mathrm{QED}$ correction

$$
R_{11}\left(\hat{Q}^{2}\right)=R_{11}^{0}\left(\hat{Q}^{2}\right)+\delta R_{11}\left(\hat{Q}^{2}\right)=\Pi_{0}^{0}+\delta \Pi_{0}+\hat{Q}^{2}\left(\frac{a^{0}+\delta a}{b^{0}+\delta b+\hat{Q}^{2}}+c^{0}+\delta c\right)
$$

From this, we find the following fit ansatz for the $O(\alpha)$ contribution

$$
\delta R_{11}\left(\hat{Q}^{2}\right)=\delta \Pi_{0}+\hat{Q}^{2}\left(\frac{1}{b^{0}+\hat{Q}^{2}}\left[\delta a-\frac{\delta b \cdot a^{0}}{b^{0}+\hat{Q}^{2}}\right]+\delta c\right) .
$$

The results from this fits are shown in figure 3 by the solid red curve (perturbative data) and the dashed blue curve (stochastic data). The QED correction to $a_{\mu}$ obtained from the results of these fits, can be found in table 1 . 
In figure 4 the ratio of the statistical errors on the QED correction to the HVP from the perturbative over the stochastic method is shown. For both data sets, we have scaled the errors with $\sqrt{\# \text { inversions }}$ to obtain an equal cost comparison. We show results for the single- $\mu$ (closed symbols) and summed- $\mu$ (open symbols) insertion each for up (purple squares) and strange (blue triangles) quarks. We find this ratio of errors to be about $1.5-2$, indicating, the we find the statistical error from the stochastic method to be a factor $1.5-2$ smaller than the statistical error from the perturbative method at the same numerical cost.

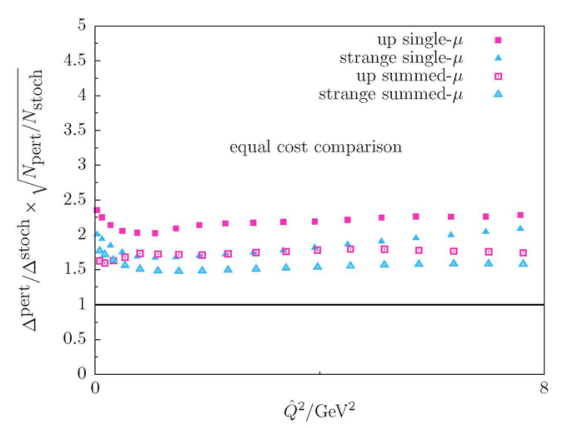

Figure 4. Comparison of statistical errors from the stochastic and the perturbative method at equal cost.

\subsection{Results - QED corrections to $Z_{V}$}

An additional QED correction to the HVP arises from the QED correction $\delta Z_{V}$ to the multiplicative renormalisation of the local vector current. $Z_{V}$ can be determined from the ratio of the local-conserved $C^{l c}$ and the local-local $C^{l l}$ two-point function

$$
Z_{V}=\frac{C^{l c}(t)}{C^{l l}(t)}=\frac{C_{0}^{l c}(t)+\delta C^{l c}(t)}{C_{0}^{l l}(t)+\delta C^{l l}(t)}=\frac{C_{0}^{l c}(t)}{C_{0}^{l l}(t)}+\underbrace{\left(\frac{\delta C^{l c}(t)}{C_{0}^{l l}(t)}-\frac{C_{0}^{l c}(t)}{C_{0}^{l l}(t)} \frac{\delta C^{l l}(t)}{C_{0}^{l l}(t)}\right)}_{=\delta Z_{V}}+O\left(\alpha^{2}\right) .
$$

Thus, the QED correction to $Z_{V}$ can be obtained from a combination of correlation functions given in the brackets in equation (17). This is shown in figure 5 for the up quark (left) and the strange quark (right). Red square points and blue circle points denote results from the perturbative and the stochastic method, respectively. We have fitted a constant to the plateau region of the data to determine $\delta Z_{V}$. The results for the QED correction to the HVP contribution to $a_{\mu}$ from $\delta Z_{V}$ can be found in table 1 .
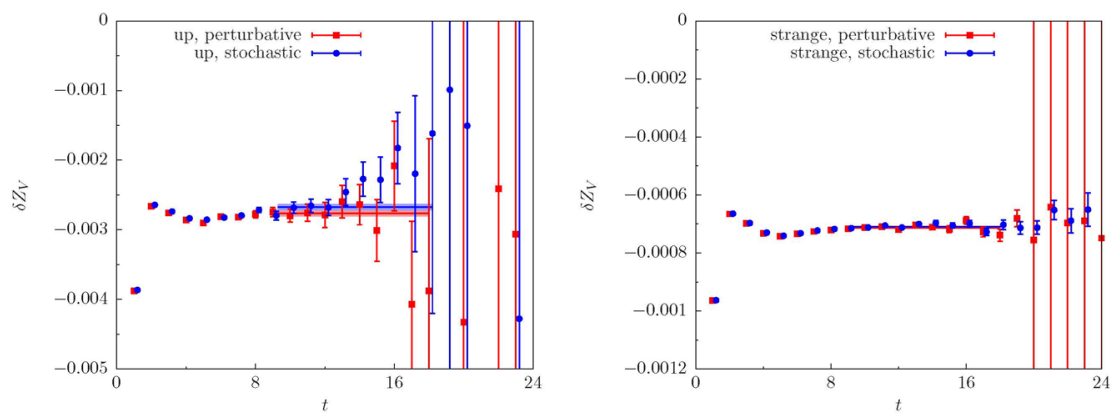

Figure 5. QED correction to $Z_{V}$ for the up quark (left) and the strange quark (right). 


\subsection{Results - strong isospin breaking corrections}

The difference of the HVP form factor calculated with the mass of the down or the up-quark is shown in figure 6. Purple squares show results from the path integral expansion (10) and green circles results from using different valence quark masses for the up and the down quark. We have fitted a Padé with a similar ansatz as for the QED corrections to the data. The results of the fits is indicated by the curves shown in figure 6 . We find $\delta_{\mathrm{s}} a_{\mu} / a_{\mu}^{u} \approx-0.9 \%$, where $\delta_{\mathrm{s}} a_{\mu}$ is the difference in $a_{\mu}$ once calculated with the down-quark mass and once with the up-quark mass. In general, a lighter quark mass results in a larger value for $a_{\mu}$ and a heavier quark mass in a smaller value.

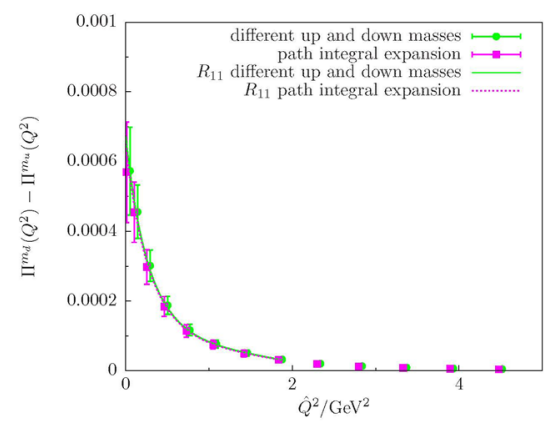

Figure 6. Difference of the HVP calculated with $m_{u}$ and $m_{d}$.

\section{Summary}

Our results for the QED corrections to the HVP contribution to $a_{\mu}$ are given in table 1 alongside results $a_{\mu}^{0}$ without QED. We give values for the two different contributions (from the correction to the vector-vector two-point function and from $Z_{V}$ ) separately. We find the QED correction to be $<1 \%$

\begin{tabular}{|c||c||c|c||c|c|}
\hline \multicolumn{1}{|c||}{$a_{\mu}^{0} \times 10^{10}$} & \multicolumn{2}{c||}{$\delta^{V} a_{\mu} \times 10^{10}$} & \multicolumn{2}{c|}{$\delta^{Z_{V}} a_{\mu} \times 10^{10}$} \\
& & stoch, $R_{11}$ & pert, $R_{11}$ & stoch & pert \\
\hline \hline$u$ & $318(11)$ & $0.65(31)$ & $0.37(33)$ & $-1.212(52)$ & $-1.249(47)$ \\
$s$ & $47.98(25)$ & $-0.0030(12)$ & $-0.0049(11)$ & $-0.04886(28)$ & $-0.04911(27)$ \\
\hline
\end{tabular}

Table 1. Summary of our results for the QED correction to $a_{\mu}$.

for the up quarks and $\approx 0.1 \%$ for strange quarks. Note, that these results are obtained at unphysical quark masses. We have not corrected our results for the QED corrections to the HVP by finite volume effects. We are currently studying these effects using lattice scalar QED [21].

We find the strong isospin correction to be $-0.9 \%$ of the isospin symmetric result. This result depends on our arbitrary choice to keep the up-quark mass fixed and increase the mass of the down quark. Only a determination at the physical point including a tuning of the up- and down-quark masses to their physical values will allow to make reliable predictions for the actual magnitude and sign of the strong isospin breaking corrections.

Acknowledgements This work has received funding from the STFC Grant ST/L000296/1, the EPSRC Centre for Doctoral Training in Next Generation Computational Modelling grant EP/L015382/1 and from the European Research Council under the European Union's Seventh Framework Programme (FP7/2007-2013) / ERC Grant agreement 279757. V.G. acknowledges partial support from the Horizon 2020 INVISIBLESPlus (H2020-MSCA-RISE-2015 -690575). P.A.B. and 
A.P. are supported in part by UK STFC grant ST/L000458/1. C.L. is supported in part by US DOE Contract \#AC-02-98CH10886(BNL) and in part through a DOE Office of Science Early Career Award. This work used the DiRAC Blue Gene Q Shared Petaflop system at the University of Edinburgh, operated by the Edinburgh Parallel Computing Centre on behalf of the STFC DiRAC HPC Facility (www.dirac.ac.uk). This equipment was funded by BIS National E-infrastructure capital grant ST/K000411/1, STFC capital grant ST/H008845/1, and STFC DiRAC Operations grants ST/K005804/1 and ST/K005790/1. DiRAC is part of the National E-Infrastructure. Computing support for this work came partially from the Lawrence Livermore National Laboratory (LLNL) Institutional Computing Grand Challenge program.

\section{References}

[1] C. Patrignani et al. (Particle Data Group), Chin. Phys. C40, 100001 (2016)

[2] M. Davier, A. Hoecker, B. Malaescu, Z. Zhang, Eur. Phys. J. C71, 1515 (2011), [Erratum: Eur. Phys. J.C72,1874(2012)], 1010.4180

[3] K. Hagiwara, R. Liao, A.D. Martin, D. Nomura, T. Teubner, J. Phys. G38, 085003 (2011), 1105.3149

[4] F. Burger, X. Feng, G. Hotzel, K. Jansen, M. Petschlies, D.B. Renner (ETM), JHEP 02, 099 (2014), 1308.4327

[5] G. Bali, G. Endrődi, Phys. Rev. D92, 054506 (2015), 1506.08638

[6] B. Chakraborty, C.T.H. Davies, P.G. de Oliviera, J. Koponen, G.P. Lepage, R.S. Van de Water, Phys. Rev. D96, 034516 (2017), 1601.03071

[7] S. Borsanyi, Z. Fodor, T. Kawanai, S. Krieg, L. Lellouch, R. Malak, K. Miura, K.K. Szabo, C. Torrero, B. Toth (2016), 1612.02364

[8] M. Della Morte, A. Francis, V. Gülpers, G. Herdoíza, G. von Hippel, H. Horch, B. Jäger, H.B. Meyer, A. Nyffeler, H. Wittig, JHEP 10, 020 (2017), 1705 . 01775

[9] P. Boyle, V. Gülpers, J. Harrison, A. Jüttner, A. Portelli, C. Sachrajda, Electromagnetic Corrections to Meson Masses and the HVP, in Proceedings, 34th International Symposium on Lattice Field Theory (Lattice 2016): Southampton, UK, July 24-30, 2016 (2016), 1612.05962

[10] P. Boyle, V. Gülpers, J. Harrison, A. Jüttner, C. Lehner, A. Portelli, C.T. Sachrajda, JHEP 09, 153 (2017), 1706.05293

[11] D. Giusti, V. Lubicz, G. Martinelli, F. Sanfilippo, S. Simula (2017), 1707.03019

[12] A. Duncan, E. Eichten, H. Thacker, Phys. Rev. Lett. 76, 3894 (1996), hep-lat/9602005

[13] G.M. de Divitiis, R. Frezzotti, V. Lubicz, G. Martinelli, R. Petronzio, G.C. Rossi, F. Sanfilippo, S. Simula, N. Tantalo, Phys. Rev. D87, 114505 (2013), 1303.4896

[14] M. Hayakawa, S. Uno, Prog. Theor. Phys. 120, 413 (2008), 0804. 2044

[15] T. Blum, T. Doi, M. Hayakawa, T. Izubuchi, N. Yamada, Phys. Rev. D76, 114508 (2007), 0708.0484

[16] Z. Fodor, C. Hoelbling, S. Krieg, L. Lellouch, T. Lippert, A. Portelli, A. Sastre, K.K. Szabo, L. Varnhorst, Phys. Rev. Lett. 117, 082001 (2016), 1604.07112

[17] G.M. de Divitiis et al., JHEP 04, 124 (2012), 1110. 6294

[18] D. Bernecker, H.B. Meyer, Eur. Phys. J. A47, 148 (2011), 1107.4388

[19] C. Allton et al. (RBC-UKQCD), Phys. Rev. D78, 114509 (2008), 0804.0473

[20] T. Blum et al., Phys. Rev. D93, 074505 (2016), 1411.7017

[21] J. Harrison et al., Numerical investigation of finite-volume effects for the HVP, in 35th International Symposium on Lattice Field Theory (Lattice 2017) Granada, Spain, June 18-24, 2017 (2017) 